\title{
Evaluation of two-dimensional strain rate imaging on left ventricular long- axis systolic function in patients with slow coronary flow.
}

\author{
Xiuxiu Fu ${ }^{1}$, Zhibin Wang ${ }^{*}$, Zuoyuan Chen ${ }^{2}$, Meixin Liu ${ }^{1}$, Wugang Wang', Junfang Li ${ }^{1}$, Yong Li ${ }^{1}$ \\ ${ }^{1}$ Department of Cardiac Ultrasound, the Affiliated Hospital of Qingdao University, Qingdao, China \\ ${ }^{2}$ Department of Cardiology, the Affiliated Hospital of Qingdao University, Qingdao, China
}

\begin{abstract}
Objective: To evaluate the value of two-dimensional strain rate imaging (STE) in detecting the alteration of regionally left ventricular long-axis systolic function in patients with slow coronary flow (SCF).

Methods: 24 patients who were with SCF but without significant coronary artery stenosis in LAD were included in left anterior descending artery (LAD) group, and 15 patients with slow flow in right coronary artery (RCA) were included in RCA group, and 20 patients who were without significant coronary stenosis or abnormal corrected thrombolysis in myocardial infarction frame count (CTFC) were included in control group. CTFC was carried on 3 groups. Peak systolic strain rate (PSRs) were measured at basal, middle and apical segments in left ventricular walls, including the septal, lateral, inferior and anterior.

Results: CTFC of left anterior descending coronary artery blood flow $(39.88 \pm 7.48)$ was significantly higher in LAD group than control group $(19.84 \pm 5.91, \mathrm{P}<0.01)$. CTFC of right coronary artery blood flow $(23.78 \pm 8.15)$ was significantly higher in RCA group than control group $(21.55 \pm 8.12, P<0.01)$. PSRs of anterior wall in LAD group and interior wall in RCA group were significantly lower than that in control group $(\mathbf{P}<\mathbf{0 . 0 1})$. Linear regression showed that the CTFC of left anterior descending artery was negatively correlated with the PSRs of anterior wall $(\mathrm{r}=-\mathbf{0 . 5 5}, \mathrm{P}<0.01)$, as well as the CTFC of right coronary artery and the PSRs of interior wall in RCA group $(-0.86, \mathbf{P}<0.01)$.

Conclusions: STE can evaluate the regional left ventricular long-axis systolic function in the patients with SCF.
\end{abstract}

Keywords: Echocardiography, Slow coronary flow, Two-dimensional strain rate imaging, Long-axis function.

\section{Introduction}

Slow coronary flow (SCF) shows many symptoms including coronary artery ectasia, coronarospasm, thrombolysis, myocardiosis, valvular heart disease (VHD), connective tissue disease (CTD) and so on. There is no obvious coronary artery disease besides that blood perfusion in arteriography delayed. SCF was common in coronary artery arteriography, in which its mechanisms were related to impaired endothelial function, microcirculation dysfunction, and inflammatory reaction and so on. Many previous researches mainly focus on the relationship between SCF and myocardial ischemia by studying metabolic indexes or abnormal heart muscle perfusion, and few studies quantitatively analyze the regionally left ventricular myocardial function using echocardiogram in patients with SCF.

Strain rate imaging of two-dimensional echocardiography (STE) was a quantitative analysis of echocardiogram, which is used to evaluate regionally myocardial function with high accuracy. By quantitative analysis of regional ischemic myocardium, STE could provide early diagnosis and therapy for coronary heart disease in clinic with direct evidences. Our study was to investigate whether the STE could be used to evaluate regionally left ventricular myocardial function in patients with SCF.

\section{Methods and Materials}

\section{Patients}

39 patients were selected, and they were received coronary arteriography and thrombolysis in myocardial infarction (TIMI), and diagnosed as SCF but without significant coronary artery stenosis, which were from Department of Cardiology of the Affiliated Hospital of Medical College (Qingdao University, Qingdao, China) between September 2011 and March 2015. Among them, 24 patients were with SCF in left anterior descending artery (LAD) but without significant coronary artery stenosis, and classified as LAD group, including 14 males and 10 females aged 40 to 65, average age $54 \pm 7.15$. Patients were classified as RCA group who were with slow flow in right coronary artery (RCA), including 9 
males and 6 females aged 44 to 63 , average age $51 \pm 8.20$. And patients were classified as control group who were without significant coronary stenosis or abnormal CTFC (corrected thrombolysis in myocardial infarction frame count), including 12 males and 8 females aged 39 to 58 , average age $49 \pm 9$. The patients were excluded who with cardiomyopathy, diabetes, VHD, heart failure, autoimmune disease (AID), tumors, serious dysfunction liver and kidney insufficiency and so on. The patients were also excluded in the control group who were received X-ray, cardiogram, and echocardiogram and so on, and diagnosed as sinus heart rate.

SCF diagnosing criteria: Corrected TIMI frame count (CTFC) was used to evaluate the blood flow rate in coronary angiography; images were collected at a rate of $30 \mathrm{frame} / \mathrm{s}$ at the beginning of contrast agent filling, the one coronary artery to distally labelling branches of vessel at the first time; for the longer LAD, the value of TIMI/1.7 was used to correct.

According to Gibson et al. [1], the standard CTFC was as following: the frame of LAD was $36.2 \pm 2.6$, which was corrected as $21.1 \pm 1.5$; the circumflex branch (LCX) was 22.2 \pm 4.1 ; the RCA was $20.4 \pm 3.0$; the SCF was confirmed as the above two normal standard deviation.

\section{Methods}

The working conditions were as following: GE Vivid 7 color Doppler echocardiography, M3S probe, 1.5 4.0 MHz, frame of 40 90/s. And the EchoPAC working station with 2D speckle tracking and strain rate imaging were used.

2D-Ultrasonography images collection: The patients stayed at left-lateral position with steady breath; the images were collected at the time holding breath at the end of breathing out, as well as cardiogram; the images of 3 cardiac cycle were recorded and analyzed, and the average value was obtained.

Parameter measurement by two-dimensional echocardiography (TDE): Left ventricular long axis was measured by standard; left ventricular end diastolic diameter (LVDd) and left ventricular end systolic diameter (LVSd) were measured. The apical four-chamber was measured by pulse Doppler; the height of the early diastolic flow velocity peak (E) and the height of the late diastolic peak (A) were measured and the value of E/A was obtained; left ventricular ejection fraction (LVEF) was calculated by biplane Simpson's.

Parameter analysis by two-dimensional strain rate (SRs): The apical four-chamber and apical two-chamber were analyzed by the two-dimensional model; dynamic images in 3 cardiac cycle were collected and analyzed. By GE EchoPAC software, the analysis system was started at end-systole (ESV) for basal, middle and apical segment in left ventricular walls, including the septal, lateral, inferior and anterior. Longitudinal peak value SRs in systole was obtained from each myocardial segment. The average of wall at the same side was taken as the peak systolic strain rate (PSRs).

\section{Statistical analysis}

All the data were analyzed by SPSS 17.0 software. The data was presented as $\overline{\mathrm{x}} \pm \mathrm{s}$. Comparison between groups was analyzed using one way ANOVA. Multiple comparisons were analyzed using LSD-q test. The correlations were analyzed using linear correlation analysis. $\mathrm{P}<0.05$ was considered that the difference had statistical significance.

\section{Results}

\section{Comparisons between clinical characteristics and measurement data by TDE}

In LAD and RCA groups, the age, heart rate, LVDd, LVSd, E, A, E/A and LVEF had no significant difference compared with control group (Table 1).

Table 1. Clinical characteristics and ultrasound parameters comparisons among LAD, RCA and control groups $(\bar{x} \pm s)$.

\begin{tabular}{|c|c|c|c|c|c|c|c|c|c|}
\hline Groups & Cases & Age (y) & $\begin{array}{l}\text { Heart rate (times/ } \\
\text { min) }\end{array}$ & LVDd (cm) & LVSd (cm) & $E(m / s)$ & $A(m / s)$ & E/A & EF (\%) \\
\hline LAD & 24 & $54 \pm 7$ & $71 \pm 8$ & $4.39 \pm 0.26$ & $2.89 \pm 0.18$ & $0.67 \pm 0.25$ & $0.76 \pm 0.22$ & $0.94 \pm 0.31$ & $66.7 \pm 5.1$ \\
\hline $\mathrm{RCA}$ & 19 & $51 \pm 8$ & $72 \pm 9$ & $4.41 \pm 0.37$ & $2.79 \pm 0.21$ & $0.69 \pm 0.18$ & $0.78 \pm 0.21$ & $0.96 \pm 0.29$ & $68.2 \pm 7.2$ \\
\hline Control & 20 & $49 \pm 6$ & $74 \pm 10$ & $4.32 \pm 0.39$ & $2.87 \pm 0.17$ & $0.77 \pm 0.19$ & $0.68 \pm 0.19$ & $1.07 \pm 0.38$ & $65.6 \pm 8.6$ \\
\hline $\mathrm{F}$ & & 0.83 & 0.62 & 0.39 & 1.64 & 1.3 & 1.3 & 1.18 & 0.68 \\
\hline$P$ & & 0.07 & 0.54 & 0.68 & 0.2 & 0.28 & 0.27 & 0.31 & 0.51 \\
\hline
\end{tabular}

Note: LVDd: Left Ventricular End Diastolic diameter; LVSd: Left Ventricular End Systolic diameter; E: The height of the early diastolic flow velocity peak; A: The height of the late diastolic peak; EF: Left Ventricular Ejection Fraction; LAD: Left Anterior Descending Artery; RCA: Right Coronary Artery.

\section{Comparison of CTFC}

CTFC of left anterior descending coronary artery blood flow was higher in LAD group $(39.88 \pm 7.48)$ than control group (19.84 \pm 5.91$)$, significantly $(\mathrm{P}<0.01)$. CTFC of right coronary artery blood flow was higher in RCA group $(23.78 \pm 8.15)$ than control group (21.55 \pm 8.12$)$, significantly $(\mathrm{P}<0.01)$ (Table 2$)$.

Table 2. Frame value of CTFC comparison among $L A D, R C A$ and control groups $(\bar{x} \pm s)$. 
Evaluation of two-dimensional strain rate imaging on left ventricular long-axis systolic function in patients with slow coronary flow

\begin{tabular}{llll}
\hline Groups & Cases & LAD & RCA \\
\hline LAD & 24 & $39.88 \pm 7.48^{\Delta}$ & $23.78 \pm 8.15$ \\
\hline RCA & 19 & $22.51 \pm 6.73^{*}$ & $41.74 \pm 6.29^{*}$ \\
\hline Control & 20 & $19.84 \pm 5.91^{*}$ & $21.55 \pm 8.12^{\Delta}$ \\
\hline F & & 57.34 & 41.5 \\
\hline P & & 0 & 0 \\
\hline
\end{tabular}

Note: LAD: Left Anterior Descending artery; RCA: Right Coronary Artery; *Comparing to LAD group, $\mathrm{P}<0.01 ;{ }^{\circ}$ Comparing to RCA group, $\mathrm{P}<0.01$.

\section{Comparison of PSRs}

PSRs were significantly lower in anterior wall in LAD group and interior wall in RCA group than control group, significantly $(\mathrm{P}<0.01, \mathrm{P}<0.01)$ (Table 3, Figure 1 and Figure 2).

Table 3. PSRs comparisons among LAD, RCA and control groups $\left(\mathrm{s}^{-1}\right.$, $\bar{x} \pm s)$.

\begin{tabular}{llllll}
\hline Groups & Cases & Septal & Lateral & Inferior & Anterior \\
\hline LCA & 24 & $1.76 \pm 0.23$ & $1.91 \pm 0.21$ & $2.05 \pm 0.18^{\Delta}$ & $1.23 \pm 0.26^{\Delta}$ \\
\hline RCA & 19 & $1.72 \pm 0.11$ & $1.95 \pm 0.23$ & $1.30 \pm 0.21^{*}$ & $1.90 \pm 0.20^{*}$ \\
\hline Control & 20 & $1.80 \pm 0.19$ & $2.06 \pm 0.20$ & $2.17 \pm 0.24^{\Delta}$ & $1.89 \pm 0.18^{*}$ \\
\hline F & & 1.38 & 2.8 & 99.6 & 68.18 \\
\hline P & 0.26 & 0.07 & 0 & 0 \\
\hline
\end{tabular}

Note: LAD: Left Anterior Descending artery; RCA: Right Coronary Artery; ${ }^{*}$ Comparing to LAD group, $\mathrm{P}<0.01 ;{ }^{\triangle}$ Comparing to RCA group, $\mathrm{P}<0.01$.
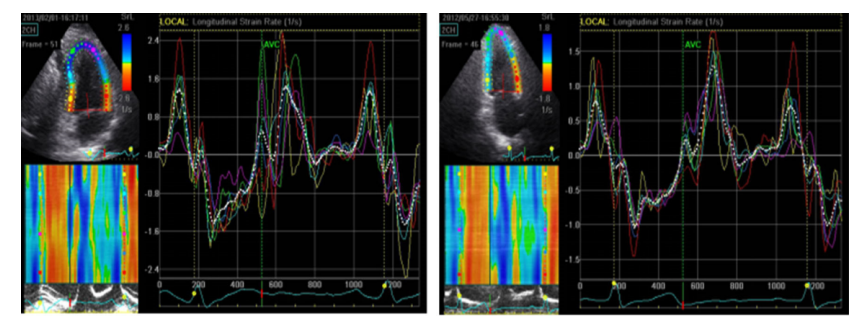

Figure 1. Comparison of the curve diagram of two-dimensional strain rate images in left ventricular anterior wall between $L A D$ and control groups. The upper was LAD group (PSRs=1.13 $\left.\mathrm{s}^{-1}\right)$, and the lower was the control $\left(P S R s=1.50 \mathrm{~s}^{-1}\right)$.
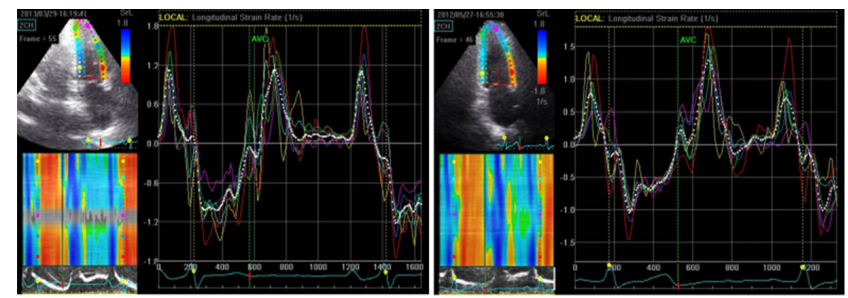

Figure 2. Comparison of the curve diagram of two-dimensional strain rate images in left ventricular anterior wall between $R C A$ and control groups. The upper was RCA group (PSRs $\left.=1.38 \mathrm{~s}^{-1}\right)$, and the lower was control group $\left(P S R s=1.53 \mathrm{~s}^{-1}\right)$.

\section{Co-relationship between PSRs and CTFC}

Linear regression showed that the CTFC of left anterior descending artery had negative correlation with the PSRs of anterior wall $(\mathrm{r}=-0.55, \mathrm{P}<0.01)$, as well as the CTFC of right coronary artery and the PSRs of interior wall in RCA group $(-0.86, \mathrm{P}<0.01)$.

\section{Discussion}

SCF was common in coronary artery arteriography, which drew more and more attentions of specialists in angiocardiopathy field. Tambe et al. [2] firstly reported the phenomenon of SCF, while Mangieri et al. [3] showed that the detection rate of SCF was $7 \%$. SCF had many clinical characteristics including chest discomfort or angina pectoris, while its mechanism is unclear now. Many studies focused on the relationship between it and the abnormal function of coronary microcirculation. Some reports showed that patients with SCF showed abnormal small vessels detected by biopsy of ventricular and myocardial tissue pathology, including thickening vascular endothelial, damaged capillary network, decreasing vessel diameter and so on, which were all caused by edema [3]. SCF would result in the ischemia, edema, even necrosis of cardiac muscle cells at the diseased vessel area, presenting ischemia myocardial with angina pectoris, Acute Coronary Syndrome (ACS) and so on. Some scientists considered that SCF was the new type of coronary syndrome [4]. Therefore, the patients with SCF should receive more attentions on the early changes of regional left ventricular myocardial function.

STE was a new quantitative analysis method of echocardiography to evaluate myocardial function with higher accuracy. The moving information of the regionally cardiac tissue was tracked by myocardial contrast speckle using the two-dimensional model. Comparing to Doppler imaging, STE had less effects on heart displacement or retraction of adjacent tissues [5-7]. But, due to no dependence of angle and high repeatability, it could be used to evaluate the systolic and diastolic dysfunction in regional myocardial function with higher accuracy. STE provided early diagnosis and therapy for clinical coronary heart disease with direct evidences [8-10].

At the early stage of ischemia myocardial, it was the first stage changes of sub-endocardial myocardial function, mainly in vertically sub-endocardial cardiac muscle fibers. Therefore, myocardial longitudinal systolic strain rate was the sensitive index in ischemia myocardial, which represented the maximum deformation degree of systole and the contractibility. Recently, the left ventricular longitudinal strain were reported that its significance in the early detection of ischemia myocardial dysfunction in sub-endocardial myocardial, as well as the sensitive index of myocardial contractility of left ventricle [11-14].

The PSRs was used as the quantitative analysis index of SCF in this study, and we found that, comparing with control group, the PSRs decreased in LAD and RCA group, but there was no significant difference in age, heart rate, LVDd, LVSd, E, A, 
E/A or LVEF. These results showed that there was no abnormal change in the systolic and diastolic function of the patients with SCF, but the early systolic function damaged at a certain level in diseased regional left ventricle vessel.

Dogan et al. [12] detected the peak diastolic flow velocity in 20 cases with SCF and found that it decreased compared with healthy people, which showed ischemia myocardial and abnormal perfusion in the patients with SCF. By detections of fractional flow reserve (FFR) measurement and intravascular ultrasound, SCF might be induced by the atherosclerotic plaque (AP) scattered in the vessel, and result in the increase of sub-endocardial coronary artery resistance. Another hypothesis considered that SCF was the early stage of coronary arteriosclerosis. Zekerjya et al. [15] found that the more vessels of SCF, the more obviously decrease of the whole and regional function in myocardium of left ventricle.

There were some deficiencies in this study including the small sample, the necessarily clearer two-dimensional images with higher frame in two-dimensional strain measurement, the influence on the measurement with lower frame, time-wasting, low repeatability.

In conclusion, the two-dimensional strain rate decreased in the patients with SCF by imaging systolic function of left ventricular long-axis, which indicated that the SCF vessel had relationship with the damage of the regional myocardial contractile function and the myocardia ischemic change.

\section{References}

1. Gibson CM, Cannon CP, Daley WL, Dodge JT, Alexander B, Marble SJ, McCabe CH, Raymond L, Fortin T, Poole WK, Braunwald E. TIMI frame count: a quantitative method of assessing coronary artery flow. Circulation 1996; 92: 879-888.

2. Tambe AA, Demany MA, Zimmerman HA, Mascarenhas E. Andian pectoris and slow flow velocity of dye in coronary arteries new andiographic finding. Am Heart J 1972; 84: 66-71.

3. Mangieri E, Macchiarelli G, Ciavolella M, Barillà F, Avella A, Martinotti A, Dell'Italia LJ, Scibilia G, Motta P, Campa PP. Slow coronary flow: clinical and histopathological features in patients with otherwise normal epicardial coronary arteries. Cathet Cardiovasc Diang 1996; 37: 375-381.

4. Li JJ, Wu YJ, Qin XW. Should slow coronary flow be considered as a coronary syndrome? Med Hypotheses 2006; 66: 953-956.

5. Gollou, Abbas R, Noradin G. A new feature selection and hybrid forecast engine for day-ahead price forecasting of electricity markets. J Intelligent Fuzzy Systems Preprint 2017; 1-15.

6. Razmjooy, Navid, Mehdi R, Noradin G. Imperialist competitive algorithm-based optimization of neuro-fuzzy system parameters for automatic red-eye removal. Int $\mathrm{J}$ Fuzzy Systems 1-13.

7. Ebrahimian H. Distributed diode single-balanced mixer using defected and protruded structures for Doppler radar applications. Appl Comput Electromagnetics Soc J 2015.

8. Parsian, Ali, Mehdi R, Noradin G. A hybrid neural network-gray wolf optimization algorithm for melanoma detection. Biomed Res 2017.

9. Ghadimi, Noradin, Mohammad O. A novel design of low power rectenna for wireless sensor and RFID applications. Wireless Personal Communications 2014.

10. Jalili, Aref, Noradin G. Hybrid harmony search algorithm and fuzzy mechanism for solving congestion management problem in an electricity market. Complexity 2016; 90-98.

11. Greenberg NL, Firstenberg MS, Castro PL, Main M, Travaglini A, Odabashian JA, Drinko JK, Rodriguez LL, Thomas JD, Garcia MJ. Doppler-derived myocardial systolic strain rate is a strong index of left ventricular contractility. Circulation 2002; 105: 99-105.

12. Erdogan D, Caliskan M, Gullu H, Sezgin AT, Yildirir A and Muderrisoglu H. Coronary flow reserve is impaired in patients with slow coronary flow. Atherosclerosis 2007; 191: 168-174.

13. Nurkalem Z, Gorgulu S, Uslu N, Orhan AL, Alper AT, Erer B, Zencirci E, Aksu H, Eren M. Longitudinal left ventricular systolic function is impaired in patients with coronary slow flow. Int J Cardiovasc Imaging 2009; 25: 25-32.

14. Teske AJ, De Boeck BW, Olimulder M, Prakken NH, Doevendans PA, Cramer MJ. Echocardiographic assessment of regional right ventricular function. A head to head comparison between 2D-strain and tissue Doppler derived strain analysis. J Am Soc Echocardiogr 2007; 21: 275-283.

\section{*Correspondence to}

Zhibin Wang

Department of Cardiac Ultrasound

The Affiliated Hospital of Qingdao University

Qingdao

China 